\title{
riccafd
}

Revista Iberoamericana de Ciencias de la Actividad Física y el Deporte

\section{RELACIÓN ENTRE CONDICIÓN FÍSICA Y LESIONES MUSCULÓ-ESQUELÉTICAS EN ESTUDIANTES DE MÚSICA} RELATIONSHIP BETWEEN PHYSICAL CONDITION AND MUSCLESKELETAL INJURIES IN MUSIC STUDENTS

\author{
Tovar Torres, Hernán Gilberto. \\ Hernán Gilberto Tovar Torres. Profesor de tiempo completo Universidad del Tolima (Colombia), \\ hgtovar@ut.edu.co
}

Código UNESCO: 2402.06 Fisiología del ejercicio

Clasificación Consejo de Europa: 6. Fisiología del ejercicio

Recibido el 2 de noviembre de 2017

Aceptado el 13 de febrero de 2018

Correspondencia:

Hernán Gilberto Tovar Torres

hgtovar@ut.edu.co

DOI: http://dx.doi.org/10.24310/riccafd.2018.v7i1.4856

Agradecimientos: Ios autores agradecen a la universidad del Tolima por la beca de estudio que permitió esta investigación y a los estudiantes de música participantes de este estudio.

\section{RESUMEN}

Existen evidencias científicas de que la condición física es un marcador biológico de la salud. El objetivo fue establecer la relación entre el nivel de condición física y la presencia de lesiones musculó-esqueléticas derivadas de la interpretación en estudiantes de carreras de música. Diseño descriptivo transversal donde se evaluó la condición física y las lesiones musculóesqueléticas derivadas de la interpretación mediante un cuestionario ad hoc, a una muestra de 300 estudiantes entre 17 y 34 años. La percepción del estado de condición física actual es percibida en criterios positivos en cuatro de cada diez. Las lesiones musculo-esqueléticas se presentaron en uno de cada dos estudiantes. El análisis relacional a través del chi-cuadrado mostró que la percepción de la condición física y las Lesiones Musculó-esqueléticas derivadas de la Interpretación (LME-I) se relacionan de modo altamente significativo. Por tanto, se concluye la necesidad de fortalecer y mejorar la condición física de este colectivo como factor de prevención para las lesiones musculó-esqueléticas derivadas de la interpretación.

Palabras clave: lesiones musculó-esqueléticas, condición física, músicos. 


\section{ABSTRACT}

There is scientific evidence that physical fitness is a biological marker of health. The objective was to establish the relationship between the level of physical condition and the presence of musculo-skeletal injuries derived from the performance in students of music races. Descriptive cross-sectional design where the physical condition and musculoskeletal lesions derived from the interpretation were evaluated through an ad hoc questionnaire, to a sample of 300 students between 17 and 34 years old. The perception of the present state of physical condition is perceived in positive criteria in four out of ten. Musculoskeletal injuries occurred in one in two students. The relational analysis through the chi-square showed that the perception of the physical condition and the LME-I are related in a highly significant way. Therefore, we conclude the need to strengthen and improve the physical condition of this group as a prevention factor for musculoskeletal injuries derived from the interpretation.

Keywords: Musculoskeletal injuries, physical condition, musicians.

\section{INTRODUCCIÓN}

Las condiciones de singularidad de trabajo y/o estudio que enfrentan a diario las personas que se dedican a la música, estos es, músicos en formación, profesionales y/o semiprofesionales, según la literatura especializada se exteriorizan principalmente en dos aspectos. En primer lugar, en los altos niveles de dedicación que requiere su actividad laboral y/o estudio en las diversas etapas de la carrera musical (formación universitaria y profesionalidad), y que, se manifiestan en un obligatorio entrenamiento diario repetitivo y que se hace necesario para alcanzar y/o mantener las destrezas que exige su profesión (rendimiento académico/musical). En segundo lugar, el alto grado de exigencia físico-mental requerido por las posturas forzadas y los movimientos repetidos de la actividad musical y la carga psicológica, aunque, a simple vista, no se perciba del todo como un trabajo/estudio de máximo esfuerzo para el músico - si los es. Dichos argumentos han llevado a la frecuente comparación del músico con deportistas de rendimiento ${ }^{1,2}$ y 3 . En este escenario de formación universitaria y/o trabajo profesional singular, que inicia desde la etapa de estudiante en formación y continúa en la vida profesional hasta la jubilación, ha llevado a que esta formación profesional tanto de experto como estudiante, se plantee como una actividad $y / u$ oficio laboral asociado a riesgos precisos de salud. Esto se complementa con las diversas investigaciones publicadas especialmente en la revista «Medical problems of professional artist», en las que coinciden los altos porcentajes de problemas de salud biopsicosocial entre los que destacan las lesiones musculo-esqueléticas derivadas de la interpretación musical (LME-I) en este colectivo ${ }^{4,5}$ y $^{6}$. La búsqueda de solución a estos problemas específicos, han llevado a que en los últimos años se consideren estrategias y acciones a favor de estas cuestiones, encontrando en el marco del profesional de la música, una adecuada forma de amortiguarlas con acciones como: la educación postural del músico, utilización de elementos ergonómicos para instrumentos, enseñanzas de técnicas de calentamiento y/o estiramiento en la sesión. Asimismo, se avanza 
en la actualidad en el estudio de recomendaciones sobre ejercicio físico, deporte, actividad física complementaria y hábitos de vida que puedan afectar negativamente al potencial artístico de este colectivo.

Sin embargo, la necesidad de identificar aspectos claves en sentido de las lesiones musculó-esqueléticas derivadas de la interpretación en el músico, llevan a plantear en el presente trabajo de investigación la relación que pueden tener otros aspectos menos explorados de estas cuestiones de salud de este colectivo, tales como la condición física. Según el Colegio Americano de Medicina Deportiva (ACSM), esta se considerada como un indicador «determinante a lo largo de la vida para desarrollar y mantener la capacidad funcional que se requiere para satisfacer las demandas durante la vida y promover una salud óptima».

\section{Objetivo}

Establecer la relación entre el nivel de condición física y la presencia de lesiones musculó-esqueléticas derivadas de la interpretación en estudiantes de carreras de música.

\section{MATERIAL Y MÉTODOS}

\section{Participantes}

Estudio descriptivo transversal compuesto por una población/muestra de 300 estudiantes de carreras de música de un conservatorio colombiano (Conservatorio del Tolima), en edades comprendidas entre los 17 a 34 años (con promedio aproximado de 21 años), se incluyeron a todos los estudiantes matriculados durante el semestre b-2014, de ambos sexos y con voluntad de participar.

\section{Variables e instrumentos}

Para establecer los resultados de las variables del estudio se han tomado algunas de las variables de un cuestionario más amplio construido para valorar el ocio y la salud en este colectivo (ad hoc). En la tabla 1 se presentan los ítems referentes a los trastornos musculo-esqueléticos derivados de la interpretación musical en este colectivo.

Tabla 1. Lista de lesiones musculó-esqueléticos derivados de la interpretación musical 


\begin{tabular}{|llll|}
\hline a. & Dolor, molestia, incomodidad, adormecimiento en boca, cara o mandíbula & $1 . \square$ & $2 . \square$ \\
\hline b. & Dolor, molestia, incomodidad, adormecimiento en cuello, hombros o abdomen & $1 . \square$ & $2 . \square$ \\
\hline c. & $\begin{array}{l}\text { Dolor, molestia incomodidad, adormecimiento en brazos, muñecas, manos o } \\
\text { dedos }\end{array}$ & $1 . \square$ & $2 . \square$ \\
\hline d. & Dolor, molestia, incomodidad, adormecimiento en espalda, cadera o nalgas & $1 . \square$ \\
\hline $\begin{array}{l}\text { e. } \\
\text { Dolor, molestia, incomodidad, adormecimiento en muslos, rodillas, tobillos o } \\
\text { pies }\end{array}$ & $1 . \square$ & $2 . \square$ \\
\hline
\end{tabular}

En el caso de la autopercepción de la condición física se valoró un ítem que midió el nivel actual al respecto. Con la utilización de una escala de Likert donde el valor 1 representa el mayor grado de insatisfacción y/o desacuerdo y el 5 el mayor grado de conformidad.

Tabla 2. Nivel de condición física actual

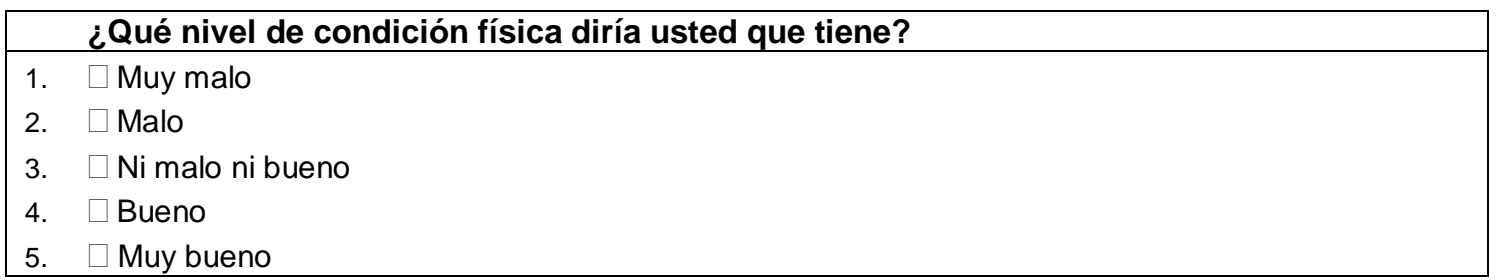

\section{Procedimiento}

Este estudio fue desarrollado durante el semestre académico B 2014 (meses de agosto a septiembre). La administración del cuestionario ad hoc, se realizó dentro de cada aula, al comienzo de cada bloque académico, es decir, antes de que el profesor diera inicio a su tema específico de clase. Para implementar el cuestionario se hizo necesario realizar dos y hasta tres visitas a las aulas con la intención de lograr implementar con el instrumento, sin excepción, a todas y todos los estudiantes del estudio. La administración fue realizada por el propio investigador, aspecto que facilitó resolver las posibles dudas, asegurar un procedimiento riguroso y garantizar el cumplimentado correcto de la trayectoria de respuesta, a tal punto de que todas las administraciones duraron menos 25 minutos y los estudiantes encuestados apenas expresaron dudas que, en todo caso, fueron resueltas por el encuestador. Se requirió el apoyo de dos colaboradores para que acompañasen el trabajo mencionado en funciones de verificación de asistencia de matriculados y reparto de los instrumentos. Estos fueron formados previamente mediante el suministro de un manual explicativo sobre la administración del cuestionario y en sesiones específicas en las que recibieron información y aclaración de dudas.

Análisis de datos

El tratamiento estadístico utilizado, una vez aplicados todos los cuestionarios, vaciada la información y grabados los datos, consistió inicialmente en el acogimiento de técnicas descriptivas. En cuanto a la estadística inferencial bivariada, ésta se realiza con un nivel de confianza del noventa y cinco por ciento $(95 \%)$ y de significancia del cinco por ciento $(5 \%)$. Se utiliza para reflejar estadísticamente la magnitud de las comparaciones y las asociaciones probabilísticas. En este sentido, las técnicas de análisis relacional de significancia se determinan así: si el valor $p$ se encuentran entre 0,01 y 0,05 
$(p=0,01$ y $p=0,05)$ se denomina significancia estadística; y si los valores $p$ son menores a 0,01 ( $p$ menor a $p=0,01$ ) se denomina alta significancia estadística. Se empleó el programa software SPSS versión 21.0.

\section{RESULTADOS}

En la tabla dos se presentan los descriptivos básicos de los trastornos musculó-esqueléticos derivados de la interpretación musical que correspondió al $77,0 \%$ La presencia de trastornos musculó-esqueléticos de brazos, antebrazos, muñecas, manos o dedos fue del $51,7 \%(n=155)$, los de espalda, cadera o nalgas en el $36,7 \%(n=110)$, seguido de los trastornos de cuello, hombros o abdomen $28,3 \%(n=85)$ entre las principales LME-I que aquejan a este colectivo.

En cuanto al género se detectó mayor presencia de estas LME-I en las mujeres $81,5 \%$ frente a los hombres $75,0 \%$. Sin embargo, la presencia de lesiones musculo-esqueléticas derivadas de la interpretación se da en porcentajes similares en las distintas zonas corporales del músico tal y como se puede ver a continuación en la tabla tres.

Tabla 3. Lesiones musculó-esqueléticas derivadas de la interpretación en las y los estudiantes de música

\begin{tabular}{|c|c|c|c|c|c|c|}
\hline & & \multirow[b]{3}{*}{$\%$} & \multicolumn{4}{|c|}{ Género } \\
\hline & \multirow[b]{2}{*}{$\mathbf{n}$} & & \multicolumn{2}{|c|}{ Hombre } & \multicolumn{2}{|c|}{ Mujer } \\
\hline & & & $\mathbf{n}$ & $\%$ & $\mathbf{n}$ & $\%$ \\
\hline Lesiones musculó-esqueléticas & 231 & $77,0 \%$ & 156 & $75,0 \%$ & 75 & $81,5 \%$ \\
\hline $\begin{array}{l}\text { Dolor, molestia incomodidad, adormecimiento } \\
\text { en brazos, muñecas, manos o dedos }\end{array}$ & 155 & $51,7 \%$ & 104 & $50,0 \%$ & 51 & $55,4 \%$ \\
\hline $\begin{array}{l}\text { Dolor, molestia, incomodidad, adormecimiento } \\
\text { en espalda, cadera o nalgas }\end{array}$ & 110 & $36,7 \%$ & 67 & $32,2 \%$ & 43 & $46,7 \%$ \\
\hline $\begin{array}{l}\text { Dolor, molestia, incomodidad, adormecimiento } \\
\text { en cuello, hombros o abdomen }\end{array}$ & 85 & $28,3 \%$ & 54 & $26,0 \%$ & 31 & $33,7 \%$ \\
\hline $\begin{array}{l}\text { Dolor, molestia, incomodidad, adormecimiento } \\
\text { en boca, cara o mandíbula }\end{array}$ & 55 & $18,3 \%$ & 34 & $16,3 \%$ & 21 & $22,8 \%$ \\
\hline $\begin{array}{l}\text { Dolor, molestia, incomodidad, adormecimiento } \\
\text { en muslos, rodillas, tobillos o pies }\end{array}$ & 35 & $11,7 \%$ & 26 & $12,5 \%$ & 9 & $9,8 \%$ \\
\hline
\end{tabular}

Con respecto al nivel de condición física en las y los estudiantes de música tal y como se puede observar en la tabla cuatro no está seguro con el $(43,7 \% ; n)$ es el porcentaje mayor con relación a este aspecto, seguido de un $(41,7 \%)$ que percibe que es buena y muy buena (saludable) y el por último, el (15\%) considera que es mala y muy mala (no saludable). La percepción de condición física saludable aumenta en los hombres $(46,7 \% ; n=97)$ al compararla con el $(30,4 \%$; $\mathrm{n}=28$ ) de las mujeres. Así mismo, la percepción negativa es mayor en las mujeres $(17,4 \% ; n=16)$ respecto a los hombres $(13,9 \% ; n=29)$. Estas diferencias entre género y condición física se confirman con la relación de significancia estadística hallada por el valor $p$. de $(p=0,019)$ entre estas variables.

Tabla 4. Percepción de la condición física en las y los estudiantes de música

\begin{tabular}{rr}
\hline \multicolumn{2}{c}{ Genero } \\
\hline RELACIÓN ENTRE CONDICIÓN FÍSICA Y LESIONES MUSCULÓ-ESQUELÉTICAS EN & 17 \\
\hline & ESTUDIANTES DE MÚSICA
\end{tabular}




\begin{tabular}{lcccccc}
\hline & $\mathbf{n}$ & $\%$ & $\mathbf{n}$ & $\%$ & $\mathbf{n}$ & $\%$ \\
\hline Muy malo & 7 & 2,3 & 5 & $2,4 \%$ & 2 & $2,2 \%$ \\
Malo & 38 & 12,7 & 24 & $11,5 \%$ & 14 & $15,5 \%$ \\
Ni malo ni bueno & 131 & 43,7 & 82 & $39,4 \%$ & 49 & $53,3 \%$ \\
Bueno & 108 & 36,0 & 84 & $40,4 \%$ & 24 & $26,1 \%$ \\
Muy bueno & 16 & 5,3 & 13 & $6,3 \%$ & 3 & $3,3 \%$ \\
Total & 300 & 100,0 & 208 & $69,3 \%$ & 92 & $30,7 \%$ \\
\hline
\end{tabular}

Con el propósito de delimitar de mejor manera la relación entre los problemas de salud derivados de la actividad musical y la condición física en las y los estudiantes de música, se analizan en la siguiente tabla cinco las distintas asociaciones realizadas entre el total de lesiones musculo-esqueléticas derivados de la interpretación musical y la percepción de condición física. En este sentido, se revela una asociación estadísticamente significativa según el valor de $\left(<\mathrm{p}={ }^{* *}, 0001\right)$, que nos confirma que la mayor proporción porcentual $(88,9 \%)$ de las y los estudiantes de carreras de música del Conservatorio del Tolima, que perciben una condición física negativa (mala y muy mala) a su vez presentan mayor presencia de lesiones musculó-esqueléticas derivadas de la interpretación musical (Si LME-I). Asímismo, se puede ver como el porcentaje más alto $(31,2 \%)$ de las y los estudiantes de música que no presentan LME-I corresponde a las y los estudiantes con una percepción de condición física más positiva (buena y muy buena).

Tabla 5. Relación entre la condición física y LME-I en las y los estudiantes de música

\begin{tabular}{|c|c|c|c|c|c|c|}
\hline & $\begin{array}{l}\text { Si lesione } \\
\text { esquelétic }\end{array}$ & $\begin{array}{l}\text { usculó- } \\
\text { LME-I) }\end{array}$ & $\begin{array}{l}\text { No lesione } \\
\text { esquelétic }\end{array}$ & $\mathrm{LME}$ & & \\
\hline Percepción condición física & $\%$ & $\mathrm{n}$ & $\%$ & $\mathrm{n}$ & $\%$ & $\mathrm{n}$ \\
\hline Malo y muy malo & $88,9 \%$ & 40 & $11,1 \%$ & 5 & $100,0 \%$ & 45 \\
\hline Ni malo ni bueno & $80,8 \%$ & 105 & $19,2 \%$ & 25 & $100,0 \%$ & 130 \\
\hline Bueno y muy bueno & $68,8 \%$ & 86 & $31,2 \%$ & 29 & $100,0 \%$ & 125 \\
\hline Total & $77,0 \%$ & 231 & $23,0 \%$ & 69 & $100,0 \%$ & 300 \\
\hline
\end{tabular}

\section{DISCUSIÓN}

Con relación a los hallazgos encontrados en esta investigación, otros estudios concuerdan con los resultados obtenidos sobre la alta presencia de lesiones musculó-esqueléticas derivados de la actividad musical en este colectivo del orden colombiano ${ }^{7}$ y e Internacional $^{9-15}$, ratificando que las LMEI se concentran en las regiones más demandadas por la interpretación musical, es decir, la región superior del cuerpo, sin distingo género ${ }^{16}$.

En cuanto, a la percepción de la condición física actual, los hallazgos de este estudio revelaron que cuatro de cada diez de las y los músicos estudiantes percibieron de manera satisfactoria este indicador. Estos resultados obtenidos difieren de otros colectivos universitarios respecto al estado de condición física que perciben las y los jóvenes estudiantes siendo más positivos (bueno/muy

18 RELACIÓN ENTRE CONDICIÓN FÍSICA Y LESIONES MUSCULÓ-ESQUELÉTICAS EN ESTUDIANTES DE MÚSICA 
bueno) ${ }^{17}$ y ${ }^{18}$. Ahora bien, en el caso de los hombres de este estudio, estos consideraron en mayor proporción una condición física saludable (buena y muy buena), con diferencias estadísticamente significativas de las mujeres; dichos hallazgos se hacen similares a los obtenidos en otros estudios con población universitaria en la que los hombres presentan una mejor condición física y una actitud más positiva ante la practica fisico-deportiva ${ }^{19}$. Similitudes que se mantienen incluso al comparar estos hallazgos con investigaciones con músicos profesionales ${ }^{20}$, dejando ver concordancias con relación a la percepción positiva de la condición física en los estudiantes universitarios y músicos profesionales.

Diversos estudios apuestan por identificar la condición física como una variable que puede incidir en la salud como factor asociado con otros factores de riesgo de la salud como el sedentarismo ${ }^{21}$ o el desempeño muscular ${ }^{22}$. En el caso de los músicos y/o estudiantes esta relación y llevada al caso de las lesiones musculó-esqueléticas derivadas de su actividad musical a la luz de la alta evidencia epidemiológica. Algunas de las evidencias científicas indican que una condición física mala o regular predispone a un riesgo alto de presentar problemas de salud, especialmente músculo-esqueléticos, en estudiantes de música ${ }^{23}$. No obstante, esta relación de la condición física como indicador de salud, requiere de más estudios para constatar los resultados obtenidos en este trabajo.

\section{CONCLUSIONES}

Con esta investigación se ha confirmado lo que la literatura especializada manifiesta en relación con la presencia de lesiones musculó-esqueléticas derivadas de su actividad musical, son las alteraciones y/o molestias que más afectan a quienes se dedican a la música en una alta proporción, tal y como ocurre con las y los estudiantes de carreras de música del Conservatorio del Tolima, Colombia, al ratificarse que las situaciones de salud musculó-esquelética están (o estuvieron) presentes en ocho de cada diez de los sujetos estudiados, confirmándose así las molestias y/o alteraciones más comunes son las LME-I de brazos, muñecas y dedos, seguidos de los de espalda, caderas y nalgas.

La conclusión más notable de este estudio respecto a la sección de la percepción de la condición física tiene que ver con la baja percepción de condición física percibida por las y los músicos estudiantes, requiere de ser fortalecida en este colectivo para que pueda influir más positivamente en su carrera, calidad de vida y salud.

Por tanto, con respecto a la salud y la relación con la condición física, este estudio ratifico que existe una prevalencia de lesiones musculó-esqueléticas derivadas de la actividad musical en las y los estudiantes de música con un nivel de condición física más negativo, convirtiendo este en un aliado clave para la aparición o ausencia de estas lesiones en este colectivo. Esto significa que existe la necesidad de modificar los hábitos de ocio en algunos aspectos para la consecución de una óptima condición física en las y los músicos desde su 
formación profesional, tales como la implementación de programas y/o estrategias que potencien la práctica de actividad física y deporte.

\section{REFERENCIAS}

1. Andrade E, e Fonseca, J. Artista-atleta: reflexões sobre a utilização do corpo na performance dos instrumentos de cordas. Per Musi: Revista de Performance Musical. Belo Horizonte: UFMG. 2000; 2(2):112-118.

2. Klein-Vogelbach S. Interpretación musical y postura corporal. Barcelona: AKAL; 2010.

3. Martín, T. Como tocar sin dolor. Tu cuerpo tu primer instrumento, Valencia: Piles; (2015).

4. Almonacid-Canseco G, Gil-Beltran I, López-Jorge, I, y Bolace-Ruiz I. Trastornos músculoesqueléticos en músicos profesionales: revisión bibliográfica. Medicina y Seguridad del trabajo. 2013; 59 (230) 124-145. [acceso 09 agosto de 2017] Disponible en: http://scielo.isciii.es/pdf/mesetra/v59n230/revision2.pdf

5. Viaño-Santasmarinas J, Díaz-Pereira P, Martínez-Vidal A. Trastornos músculoesqueléticos (TMRIs) en músicos instrumentalistas estudiantes de secundaria y universitario. Revista de Investigación en Educación. 2010; n8, (83-96). [acceso 19 septiembre de 2017] Disponible en: http://webs.uvigo.es/reined/

6. Stenger J. Lesiones musculó-esqueléticas asociadas a la interpretación musical: su comprensión y clínica. Una exploración situada en la psicología; 2015.

7. Gómez-Patiño, B, Guerrero-Luna, Y, Díaz-Fernández, Y, y Vernaza- Pinzón, P. Programas de acondicionamiento físico en músicos. Revista Iberoamericana de Ciencias de la Actividad Física y el Deporte. 2016; 5(2): 16-30.

8. Bernal A, Santana O, y Jerez G. Investigación descriptiva de los procesos laborales y los factores de riesgo presentes en las orquestas sinfónicas y filarmónicas de Colombia. Bogotá: Nuevo Rumbo; 1998.

9. Ortiz ML. Prevalencia de sintomas osteomusculares en miembros superiores y los probables factores de riesgo asociados en los estudiantes de música en una institución universitaria de Bogotá, Colombia. Revista en salud ocupacional y ambiental, Universidad del Rosario. 2014. [acceso 23 septiembre de 2017] Disponible en: http://repository.urosario.edu.co/bitstream/handle/10336/8878/516050772014. pdf? sequence $=1$ \&isAllowed $=y$. 
10. Orozco J, y Sole E. Tecnopatías del músico: prevención y tratamiento de las lesiones y enfermedades profesionales de instrumentistas y cantantes; introducción a la medicina de la danza". Barcelona: Aritza; 1996.

11. Hoppman R. Musculoskeletal problems in intrumental musicians (Vol. $2^{\circ}$ ed.). London: Singular Publishing Group; 1998.

12. Nunes D. Estrategias de prevención y tratamiento del síndrome por sobreuso en los músicos. Tesis Doctoral. Universidad de Barcelona, España; 2001.

13. Iñesta C. Demandas fisiológicas en músicos profesionales. Tesis doctoral. Oviedo, Asturias, España: Universidad de Oviedo; 2006.

14. Viaño JJ. Proyecto integral de intervención e investigación en salud y actividad física en Conservatorios de música. EE.UU. Human Movement; 2009.

15. Betancor, I. Hábitos de actividad física en músicos de orquestas sinfónicas profesionales: Un análisis empírico de ámbito internacional. Tesis Doctoral. Universidad de las Palmas de Gran Canarias, 2011.

16. Martín T, y Farías MJ. (Estrategias para promover la salud y prevenir las lesiones musculoesqueléticas en estudiantes de Conservatorio Superior de Música de Salamanca, España. Performing Art Medicine. 2013; 28(2):100-135.

17. Viaño, JJ. Trastornos musculo-esqueléticos relacionados con la interpretación musical en estudiantes instrumentistas. Epidemiología y factores de riesgo. Actividad física y deporte, hábitos de prevención y carga física. New York: Human Movement; 2009.

18. Águila, SC. Ocio, jóvenes y postmodernidad. Almería: Servicio de publicaciones Universidad de Almería; 2005.

19. Fonseca-Camacho DF, Hernández-Fonseca JM, González-Ruíz, K, TordecillaSanders A y Ramírez-Vélez R. Una mejor auto-percepción de la condición física se relaciona con menor frecuencia y componentes de síndrome metabólico en estudiantes universitarios. Nutrición Hospitalaria. 2015; 31(3): 1254-1263.

20. Pavón Lores A y Moreno Murcia JA. Actitud de los universitarios ante la práctica físico-deportiva: diferencias por géneros. Revista de psicología del deporte. 2008; 17(1): 7-23.

21. Betancor, I. Hábitos de actividad física en músicos de orquestas sinfónicas profesionales: Un análisis empírico de ámbito internacional. Tesis Doctoral. Universidad de las Palmas de Gran Canarias, 2011.

22. Prieto-Benavides DH, Correa-Bautista JE, y Ramírez-Vélez R. Niveles de actividad física, condición física y tiempo en pantallas en escolares de Bogotá, Colombia: Estudio FUPRECOL. Nutrición Hospitalaria. 2015; 32(5): 2184-2192. 
23. Rodríguez Valero FJ, Gualteros, JA, Torres JA, Umbarila Espinosa LM y Ramírez-Vélez R. Asociación entre el desempeño muscular y el bienestar físico en niños y adolescentes de Bogotá, Colombia. Nutrición Hospitalaria. 2015; 32(4): 1559-1566.

24. Viaño, JJ. Trastornos musculo-esqueléticos relacionados con la interpretación musical en estudiantes instrumentistas. Epidemiología y factores de riesgo. Actividad física y deporte, hábitos de prevención y carga física. New York: Human Movement; 2009.

Referencias totales citadas: $\mathbf{2 4}$

Referencias citadas correspondientes a la Rev lb CC Act Fis Dep: 1 